\title{
Capturing patients' daily life experiences after Total Hip Replacement
}

\author{
Juan Jimenez \\ Delft University of Technology / \\ University of Twente \\ IDE Faculty / EEMCS Faculty \\ Delft / Enschede, The Netherlands \\ J.C.JimenezGarcia@tudelft.nl
}

\author{
Natalia Romero \\ Delft University of Technology \\ Faculty of Industrial Design Engineering \\ Delft, The Netherlands \\ N.A.Romero@tudelft.nl
}

\author{
David Keyson \\ Delft University of Technology \\ Faculty of Industrial Design Engineering \\ Delft, The Netherlands \\ D.V.Keyson@tudelft.nl
}

\begin{abstract}
Context-aware technology in the home environment can provide an effective mechanism in supporting the wellbeing and autonomy of the elderly challenged by chronic diseases and physical impairments, such as osteoarthritis. Total Hip Replacement (THR) is the most common procedure to relieve both pain and functional disability experienced by elderly patients suffering from end-stage osteoarthritis, thereby improving their quality of life. The paradigm shift in healthcare on shortening in-hospital care has created an atmosphere of misinformation for both the patient and the medical staff about individual patterns of recovery, creating uncertainty about patients' recovery outcomes. This paper presents work on applying User Centred Design methods (UCD) to inform the design of a qualitative measure which will be used as a means to reduce the lack of information around the patient. The described design process elicits functional system requirements, and guides the design of a qualitative measure based on Experience Sampling Method (ESM). The ESM tool will be used to improve and expand the understanding of elderly patient's living experiences after discharge.
\end{abstract}

Keywords-context-aware systems; methods for elicitation of requirements; user centred design; experience sampling method; total hip replacement; healthcare

\section{INTRODUCTION}

Osteoarthritis (OA) is the most common joint disorder of individuals above the age of 65 [1]. Patients with OA of the hip joint experience pain, stiffness and loss of joint function. Total Hip Replacement (THR) is an effective and common solution for moderate or severe osteoarthritis. This procedure considerably reduces pain and enables patients to walk again [2]. The increasing prevalence and incidence of THR procedure in Western countries [3], due to improvements in surgical techniques, along with a greater demand for hospital beds has stimulated short-stay care. Rehabilitation programs are based on a quick discharge from the hospital, transforming the home as the main self-care environment $[4,5]$.

There are several advantages of short-stay care programs: for the hospital it represents a reduction of waiting lists and financial savings; for the patient it represents a shorter waiting time for getting the surgery. However, related studies report that an early discharge process from the hospital focuses more on the functional recovery, with little attention to the psychosocial needs for living with a new hip [2]. The short time at hospital and a quicker discharge process have decreased the time for the specialized care team to educate patients [6], sending them home without adequate educational preparation to manage their recovery [7], while increasing their own responsibility in rehabilitation.

Medical staff may deploy standardized surveys to measure patients' needs prior surgery, and functional recovery outcomes several months after discharge. Nevertheless, such questionnaires typically do not focus on the understanding of patients' experiences during the critical weeks of rehabilitation, including what physical and psychosocial patterns co-exist, and how they may evolve through time [6, 8]. Standardized questionnaires are commonly designed to document experiences in a snapshot format with high demand on patients' recall skills, while overlooking their emotional responses [7]. The lack of information on the recovery process makes it difficult to form tailored therapy after discharge [5], and to anticipate rehabilitation trajectories while the patient is at home [9].

A context-aware system to support elderly patients undergoing THR recovery at home is being developed. The system utilizes a Wireless Sensor Network (WSN) and onbody accelerometers to measure daily activity. The design is challenging from the technological as well as design perspective in terms of identifying user requirements, including motivational strategies to ensure user involvement. In order to design a support system that can provide feedback in relation to user enquires and activity measurements, knowledge relating to typical needs and emotional transitions during the recovery period is needed.

To gain insight into the patients' recovery process, firstly User Centred Design (UCD) methods were applied with the stakeholders to explore the context of patients having undergone THR surgery, and to define the functional requirements of a system for measuring physical activity levels. It became apparent early on in the process that the insights collected via the UCD methods were not sufficiently sensitive to gain insights into the dynamics of the recovery process and support needs of the patients. Therefore it was necessary to design a qualitative tool that allows collecting insights of participant's experience during the recovery process. 
This paper reports on an early usability test and present the final concept of a self-reporting tool that is currently being tested in the field to gain insights into the THR surgery recovery process. The insights in-turn will be used to develop a motivationally rich system for sharing experiences between THR patients and stakeholders. Inputs relating to physical activity levels will also be made available for sharing

\section{THE APPLICATION OF USER CENTRED DESIGN METHODS}

The first step towards understanding the physical and mental implications of THR surgery and the determinants of the physical function recovery process was to apply UCD methods with stakeholders in small groups and individual sessions, involving engineers, designers, physiotherapists and patients. The methods used were applied as follows: First, two workshops were conducted with engineers and physiotherapists. Following, scenarios as stories that describe what people might do and experience as they tray to make use of the system, guided conversations towards the definition of possible functional requirements. Next, qualitative interviews were applied with a physiotherapist and an elderly patient to capture their own perspective about THR. This protocol of multiple UCD methods over repeated sessions led to a range of insights, and a body of knowledge every time. A total of 15 participants were involved in the sessions ( 9 technicians and researchers working in the field of Ambient Technology; 5 physiotherapists from different private practices, mean age 35 ; and 1 patient, three weeks after surgery, age 65).

\section{A. Analysis}

The data was collected, analysed and interpreted based on the Grounded Theory (GT), as a strategy for qualitative research, generating concepts and theory from the collected data. The method supports the analyses of qualitative data by focusing on emerging patterns instead of using pre-set structures to study the data [10]. The first step consists in watching to the recorded videos, while keeping an open mind on what participants express. This approach can help the researcher gain a broader interpretation of the data. Following this step, full transcripts of the videos were made. The resulting text was organized and coded to identify possible important indicators or patterns about the rehabilitation process.

\section{B. Insights}

The rehabilitation process is most intensive during the first seven weeks following surgery. It was found that during this period seven factors vary over time, and they influence the rehabilitation process on both functional recovery and the emotional state: physical activity level, leg load, luxation, gait, stride velocity, stride length and the emotional experiences (Fig. 1). In this critical period patients are geared towards regaining mobility and independency, coming from disability to ability, while getting rid of the pain. Second, the high responsibility patients have towards their own recovery at home makes patients face an unpredictable recovery experience of self-care and self-improvement to reach the expected physical outcome. Third, due to this unpredictable experience, patients might have ambivalent emotions (e.g. being happy because of being at home again, but frustrated, not knowing what to do next).

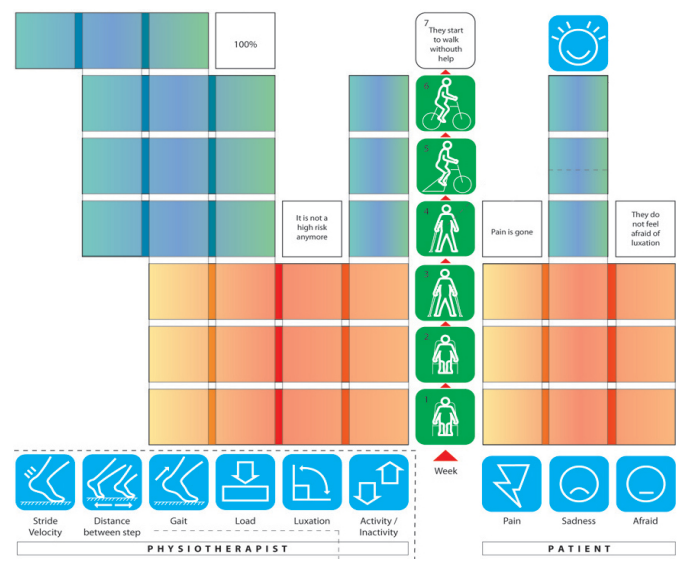

Figure 1. Overview of the 7 initial weeks of recovery. The determiants of the recovery over time: Emotional state, physical activity, luxation, leg load, gait, stride length and stride speed.

\section{DESIGNING AN EXPERIENCE SAMPLING METHOD TOOL}

In comparison to other naturalistic observation approaches, such as interviews, observation, recall surveys and diaries, Experience Sampling Method (ESM) has been shown to provide a relatively high degree of acceptance and reliability, in terms of subject recall errors [11, 12]. An experience sampling measure can be activated only when needed by triggering questions at specific moments, by means of portable computing devices, using scheduled self-report entries, and keeping a low level of user's demand of input as possible. ESM usually target the recollection of experiences, emotions or mood states regarding a particular moment in time or a recent event. An important consideration in the design of ESM studies is to trigger the questions in a low sampling rate to avoid annoying interruptions, providing engaging mechanisms to create sympathy and therefore motivate participants to selfreport.

\section{A. The initial prototype}

1) Web-app: A web-based application has been developed in HTML5 and AJAX (asynchronous javascript and XML language). Patients' inputs with the system are logged and sent to a web server for later analysis. The server administrates the schedule of the prompts as well as the inputs from the participants. Text messages and mood selections are stored in the database identified by a timestamp and type of question.

2) Touch-screen device: An iPad aims to enable simple input-data collection for elderly, eliminating the mouse barrier to a more intuitive way to interact with the device. Touchscreens have been shown to be an effective means of usersystem interaction in ubiquitous, and pervasive technologies studies for elderly [13].

3) The mood chart: The PMRI a mood capturing technique [14] that offers a pictorial representation of the Circumflex Model of Affect [15] was applied. A selection of eight moods (excited, cheerful, relaxed, calm, bored, sad, irritates, tense) have been found to be rich and easy-to-use to report moods. The tool is used in the current study to recollect moods (rather than feelings) to depict the emotional state of participant regarding their recovery, and how they see 
themselves when facing the challenges of their recovery. Since the rehabilitation process involves variations in mood, it is plausible patients experience mixed and ambivalent feelings. For instance, he/she could be anxious about possible complications, and at the same time he/she could be happy that the surgery is over with. To map this pattern, the mood chart allows the patient to select one or two moods at one time.

4) Prompting: Two types of prompts were designed. Prompt 1 asks three times a day a question regarding the patient's current mood (i.e., "How do you feel now, and why?"). Prompt 2 asks at the end of the day an overview about their mood and daily activities (i.e., "How was your day, and why?").

\section{B. Usability Test}

Three research questions were addressed: a) general use on the iPad ESM app and robustness; b) users' understanding of the PMRI tool; c) user's preference between text and voice input. This session was conducted in Enschede, The Netherlands, 2010. A total of 6 participants $(4$ men and 2 women) over the age of 60 without severe illnesses, and undergoing different stages of recovery process (no more than 1 year), attended the session. Two researchers and one physiotherapist conducted the session.

1) Session set up: Two separated rooms with two separated activities were prepared. In the "discussion room" the session was introduced and closed; participants shared their personal experiences about the recovery and they filledin the provided questionnaires. While the group were chatting in "discussion room", each participant - one after anotherwalked through the "task room" to answer the app-questions. This separate room allowed a more comfortable and independent space, simulating the home atmosphere. In this room, two researchers were present witnessing the use on the iPad. The researchers were careful not to bias respondents by supporting participants through the tasks only when they felt confused during the use of the application.. The sessions were video recorded to capture participants' opinions and experiences with the device when answering the questions (Fig. 2). The whole session lasted 120 minutes and it was conducted in Dutch. We split participants in two groups. Group A was asked to type the first prompt and voice recorded the second prompt; whereas Group B was asked to voice record the first prompt and type the second prompt.
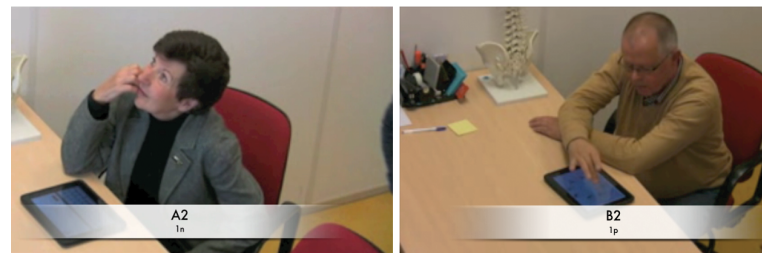

Figure 2. Tasks room. Participants are answering the questions on the iPad

2) Results: The collected data consisted in four hours of video recording, more than 30 written pages of questionnaires, and one Excel sheet of patients' inputs on the application. They were first transcript and then translated into English. Then, the data was then reviewed by one physiotherapist and two researchers to detect usability issues, and patients' opinions towards the value of this tool as a mechansim to report on the recovery process.

\section{SELF-REPORTING TOOL}

\section{A. Prompting:}

Participants seem to be confused when trying to assess and answer: "how do you feel now?" since they did not know what exactly the application was asking about, whether in general, right at the moment, or a specific situation in the immediate past. The difficulty respondents had in discerning and describing their own emotions could be attributed to the fact that individuals tend to recognize emotions as isolated ambiguous units that overlap several experiences [16]. In order to help participants, both questions and texts have to be consistent in driving participants to a more reflective process before they report an emotional state.

The final concept samples data based on a fixed interval scheduled protocol, equally spread over the day every four hours. Two types of prompts are triggered: Prompt 1 consists of an open question (How are you doing today?), and a close/open question (What is your mood at this moment? Why?) that uses the mood chart to sample the current mood states regarding the recovery; this prompt triggers three times a day (e.g. 9:00, 13:00 and 17:00). Prompt 2 consists also of an open question (How has the recovery influenced your day today?) that samples an overview of the day and a close/open question (Please indicate how your situation was today, Why?) that uses the mood chart to sample the overall mood of the day; this prompt is triggered in the evenings (e.g. 22:00). The open questions can be answered via text messages or voice recorder.

\section{B. Host character:}

The host character should receive a more predominant presence during the use of the application. She should guide the user through the different stages of the prompting and her dialogue texts should be improved, always engaging the patient to answer the questions in a friendlier way. The working assumption here is that this female character might be more familiar for the patient, improving his/her participation (for instance, she might remind a nurse or a physiotherapist). It would give a stronger sense of being "heard" by someone, adding some value to the device. This assumption should be confirmed with the final results of this study.

\section{Graphical interface:}

The application appeared to require too many screen layers before the participant actually reaches an action, (e.g. voice recording), or finishes the question. The steps should be reduced considerably (Fig. 3). A common coloured bar dialogue box reinforces the guidance for the user. A consistent colour palette should be selected to connote a more subtleclassical style. This assumption should be confirmed with participant's opinions about the tool, in further field trials with patients. 


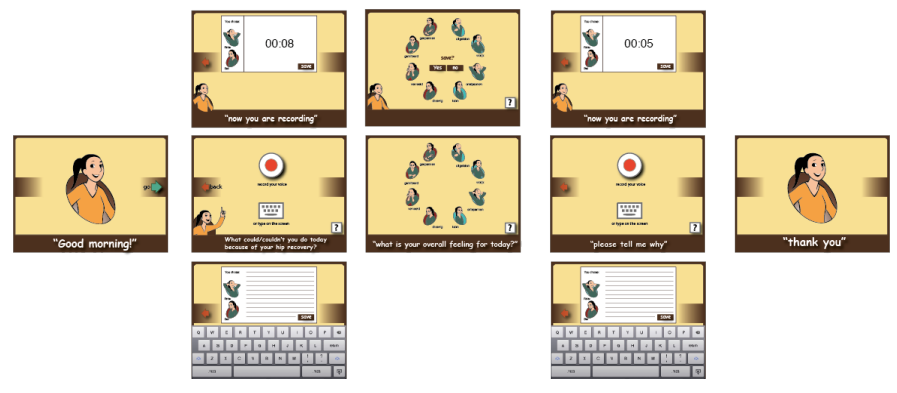

Figure 3. Prompt navigation flow (question 1).

A key finding of the studies based on the ESM system is that THR patients look for ways to share and communicate complains and questions regarding their recovery. In order to provide this "communication window", and make stronger the sense that they are heard further by the medical team, an idle state is implemented to allow participants to tell whatever they want to say, whether regarding their daily life, or something they want to ask.

\section{Touch screen input and voice recording}

Participants are willing to use the voice recorder. Elderly patients were more confident to "tell a story" about their daily activities, instead of type all through what they did.

\section{DISCUSSION}

A context-aware system can be fine-tuned to support THR patients in their rehabilitation process by means of qualitative and explorative approaches. This was possible by applying UCD methods to understand the stakeholders' needs and desires. The identification of the key factors of THR rehabilitation helped developers in an earlier phase of the development process. However, we acknowledge that even with several UCD methods, details about functional requirements regarding what kind of feedback, and how it should be communicated to the users during recovery were still insufficiently determined. Hence, an ESM tool is proposed as an objective and easily administrated assessment tool to document event of sequences in daily life, moods, and determine patients' needs regarding hip recovery and their variations over time.

\section{CONCLUSIONS AND FUTURE WORK}

In the current paper an ESM tool that aims to capture an acute view of the life of THR patients during recovery was presented. Patients can report on activities and psychosocial processes. In order to truly understand the effect of this tool in the recovery experience, as first step, two field studies with two patients in their first two weeks of recovery have been recently been conducted and are currently under analysis. The inputs to the ESM tool could ultimately be used to drive a system that provides for sharing of experiences and physical recovery levels reflected in monitored activity rates for THR patients.

\section{ACKNOWLEDGMENT}

The work presented in this paper arose from SENIOR project, an initiative within the program of economical innovation Pieken in de Delta Oost-Nederland.

\section{REFERENCES}

[1] N Arden, MC. Nevitt, "Osteoarthritis: epidemiology," Best Pract Res Clin Rheumatol, vol. 20, pp. 3-25, 2006.

[2] J. Wong, S. Wong, E. Brooks, R. H. Yabsley, "Home readiness and recovery pattern after total hip replacement," Journal of Orthopaedic Nursing, ORTHOPAEDIC NURSING, vol. 3, pp. 210-219, 1999.

[3] O. Robertsson, M. Dunbar, K. Knutson, L. Lidgren, "Past incidence and future demand for knee arthroplasty in Sweden: A report from the Swedish Knee Arthroplasty Register regarding the effect of past and future population changes on the number of arthroplasties performed," Acta Orthopaedica Scandinavica, vol. 71, pp. 376-380, 2000.

[4] Van de Aast M, Van Haastregt J, "Operation hip: a pilot research into possibilities and impossibilities of shortening of hospital stay in people who underwent a hip operation," TNO1994.

[5] M. Stevens, van den Akker-Scheek, A. Spriensma, NA. Boss, RL. Diercks, JR van Horn, "The Groningen Orthopedic Exit Strategy (GOES): a home-based support program for total hip and knee arthroplasty patients after shortened hospital stay," Patient education and counseling, vol. 54, p. 95, 2004.

[6] M. Fortina, S. Carta, D. Gambera, E. Crainz, P. Ferrata, "Recovery of physical function and patient's satisfaction after total hip replacement (THR) surgery supported by a tailored guide-book," Acta bio-medica : Atenei Parmensis, vol. 76, pp. 152-6, 2005.

[7] JM. Fielden, S. Scott, J. G. Horne, "An Investigation of Patient Satisfaction Following Discharge After Total Hip Replacement Surgery," Orthopedic nursing, vol. 22, pp. 429-436, 2003.

[8] S. Grant, W. St John, E. Patterson, "Recovery From Total Hip Replacement Surgery: "It's Not Just Physical"," Qualitative Health Research, vol. 19, pp. 1612-1620, 2009.

[9] M. Williams, M. Oberst, B. Bjorklund, S. Hughes, "Family care giving in cases of hip fracture," Rehabilitation Nursing, vol. 21, pp. 124-131, $138 \& 168,1996$

[10] J. Corbin and A. Strauss, "Grounded theory research: Procedures, canons, and evaluative criteria," Qualitative sociology, vol. 13, pp. 3-21, Jan 11990.

[11] M. Csikszentmihalyi and R. Larson, "Validity and reliability of the Experience Sampling Method," The Journal of Nervous and Mental Disease, vol. 175, pp. 526-36, 1987.

[12] J. Nawyn, SS. Intille, K. Larson, "Tools for Studying Behavior and Technology in Natural Settings," presented at the Ubiquitous computing, UbiComp 2003, Berlin, 2004.

[13] S. Consolvo and J. Towle, "Evaluating an ambient display for the home," in CHI '05: CHI '05 extended abstracts on Human factors in computing systems, New Yor, USA, 2005.

[14] M. Vastenburg, N. Romero, D. van Bel, P. Desmet, "PMRI: Development of a Pictorial Mood Reporting Instrument. Work in Progress. CHI '11 Extended Abstracts Proceedings," presented at the CHI, 2011.

[15] J.A. Russell, "A circumflex model of affect," Journal of Personality and Social Phychology, vol. 39, pp. 1161-1178, 1980.

[16] C. Saarni, The development of emotional competence: New York : Guilford Press, 1999. 